\title{
Impacto do escore MELD na sobrevida de pacientes portadores de carcinoma hepatocelular, transplantados no Brasil: revisão sistemática.
}

\section{Impact of the MELD score on the survival of hepatocellular carcinoma transplantation patients in Brazil: a systematic review.}

\author{
Marcel Vasconcellos ${ }^{1} \mathbb{0}$; Luíza Magalhães Zamith ${ }^{1}$
}

\author{
R E S U M O
}

\begin{abstract}
O objetivo do estudo foi o de analisar o valor preditivo do escore MELD (Model for End-Stage Liver Disease) na sobrevida de médio e longo prazo em pacientes portadores de carcinoma hepatocelular (CHC), transplantados no Brasil. O estudo foi registrado no PROSPERO (International Prospective Register of Systematic Reviews), sob o no 152.363. Os critérios de inclusão basearam-se nas recomendações PRISMA. A pesquisa foi realizada nos bancos de dados indexados do Lilacs, SciELO, Pubmed e Cochrane Library, e utilizou como estratégia de busca os termos MeSH: ((("Meld Score") OR "Model for End-Stage Liver Disease") AND "Hepatocellular Carcinoma") AND ("Brazil"). Foram incluídos artigos com texto completo, publicados a partir de janeiro de 2006 até outubro de 2019. A busca inicial encontrou 162 artigos. Após a leitura dos resumos e textos completos disponíveis, foram excluídos 156 artigos, totalizando seis artigos para análise qualitativa. Embora o número reduzido de artigos elegíveis tenha sido um fator limitante do estudo, nossos resultados corroboraram parcialmente aos encontrados nos EUA, Reino Unido e Irlanda. Nestes países, ao contrário do Brasil, o modelo prognóstico MELD mostrou forte associação com a sobrevida pós-transplante hepático. No entanto, a baixa capacidade preditiva do modelo em médio e longo prazo, foi similar ao nosso estudo. Configura-se a premência do desenvolvimento e validação de um modelo de sobrevida pós-transplante aos portadores de CHC, aperfeiçoando o sistema de alocação de órgãos no Brasil.
\end{abstract}

Descritores: Transplante de Fígado. Carcinoma Hepatocelular. Análise de Sobrevida.

\section{INTRODUÇÃO}

( carcinoma hepatocelular (CHC) é responsável por mais de $90 \%$ das neoplasias malignas hepáticas primárias e considerado a sexta doença maligna mais comumente diagnosticada no mundo'. Devido ao seu diagnóstico tardio, a sobrevida média dos pacientes é de aproximadamente seis a 20 meses, com sobrevida de cinco anos menor do que $12 \%{ }^{2}$.

No Brasil, $70 \%$ a $80 \%$ dos casos de CHC estão associados à cirrose secundária à infecção crônica pelos vírus B ou C³. Em 2006, uma nova política de alocação equitativa de enxertos hepáticos MELD (Model for End-Stage Liver Disease) passou a ser implantada no país com um sistema pontuação que compreende uma escala de valores de 6 a 40. Este modelo de doença hepática em estágio terminal é considerado um método bem estabelecido (AUC $=0,78-0,87$ ) como preditor de mortalidade em três meses dos pacientes que ainda se encontram na lista de espera por um órgão4.
Como indicador da gravidade da disfunção bioquímica de estágio final do receptor, o escore MELD, utiliza um cálculo logarítmico envolvendo creatinina sérica, bilirrubina e Razão Normalizada Internacional (INR), que avalia a tendência de coagulação sanguínea segundo a fórmula: [0,957 x loge (creatinina $\mathrm{mg} / \mathrm{dl})+0,378 \times$ loge (bilirrubina $\mathrm{mg} / \mathrm{dl})+1,120 \times$ loge $(\mathrm{INR})+0,643] \times 10^{4}$.

Embora portadores de CHC apresentem um menor escore MELD fisiológico, tais pacientes estão sob um risco maior de morte relacionada à progressão tumoral, assim como, remoção da lista de espera por desenvolvimento de metástases ou descompensação clínica. Desse modo, a legislação brasileira determinou que pacientes portadores de CHC, ao atenderem aos critérios de elegibilidade de Milão, obtêm uma pontuação MELD inicial de 20 pontos, que poderá progredir para 24 e 29 pontos a cada três meses, caso não tenha sido realizado o transplante hepático $(\mathrm{TH})$ ao final de seis meses $^{5}$.

1 - Centro Universitário Serra dos Órgãos (UNIFESO), Faculdade de Medicina, Teresópolis, RJ, Brasil. 
Os critérios de Milão são definidos pela presença de um nódulo maior do que $5 \mathrm{~cm}$ de diâmetro ou, no máximo, três nódulos, cada um com menos de $3 \mathrm{~cm}$ de diâmetro ${ }^{6}$. Tais critérios abreviam o tempo na lista de espera durante a evolução da doença hepática em estágio terminal7,8. A adoção de uma pontuação adicional MELDc (corrigido) a esses pacientes, reduziu o tempo na lista de espera e aumentou o número de transplantes. No entanto, o impacto do escore MELD na sobrevida desses pacientes, ainda permanece controverso $0^{9,10}$.

Há que se acrescentar, ainda, que o sistema ideal não deve definir apenas a probabilidade de morte na lista de espera, mas também prever o risco de morte após o transplante, aumentando sua utilidade ${ }^{\text {. }}$.

O objetivo desta revisão foi analisar o valor preditivo do escore MELD na sobrevida de médio e longo prazo em pacientes portadores de CHC transplantados no Brasil.

\section{MÉTODOS}

\section{Estratégia de busca}

Os critérios de análise e inclusão seguiram as recomendações PRISMA (Preferred Reporting Items for Systematic Reviews and Meta-Analyses) ${ }^{11} \mathrm{e}$ o estudo foi registrado no PROSPERO (International Prospective Register of Systematic Reviews) ${ }^{12}$ sob no 152.363 .

As bases de dados Lilacs, SciELO, Pubmed e Cochrane Library foram consultadas retrospectivamente a partir de janeiro de 2006 até outubro de 2019, utilizando como estratégia de busca os termos MeSH (Medical Subject Headings): ((("Meld Score") OR "Model for End-Stage Liver Disease") AND "Hepatocellular Carcinoma") AND ("Brazil"). A busca não fez restrição quanto ao idioma.

\section{Seleção dos estudos}

Durante a seleção dos estudos, a avaliação dos títulos e resumos identificados na busca inicial foi realizada por dois pesquisadores de forma independente e cegada, obedecendo aos seguintes critérios de inclusão e exclusão: critérios de inclusão- pacientes de ambos os sexos, idade maior do que 12 anos, com ou sem cirrose, portadores de carcinoma hepatocelular (CHC) classificados segundo os critérios de Milão; critérios de exclusão- pacientes transplantados por falência hepática aguda/hepatite fulminante e aqueles submetidos a transplantes de múltiplos órgãos, do tipo "split-liver" ou "em dominó".

\section{RESULTADOS}

\section{Estudos elegíveis}

A busca inicial nas bases de dados indexadas resultou no total de 162 artigos: 38 no Lilacs, 36 no SciELO, 20 no Pubmed e 68 na Cochrane Library. A leitura dos resumos e textos completos disponíveis levou à exclusão de 156 artigos (96,2\%). Desses, 155 artigos (95,6\%) não se enquadraram nos critérios de inclusão ou não tinham pertinência com o estudo. Um estudo $(0,6 \%)$ foi excluído por ser duplicado. Ao final, seis artigos (3,7\%) foram elegíveis para a análise qualitativa (Tabela 1) 13-18.

\section{DISCUSSÃO}

O desenvolvimento e validação de um modelo que possa prever com segurança o resultado pós-transplante com base em variáveis prétransplante, constitui-se numa tarefa extremamente complexa, dado que eventos independentes que ocorrem no período perioperatório, como rejeição do enxerto, complicações biliares, complicações vasculares e a própria habilidade da equipe cirúrgica, podem interferir no desfecho primário do estudo. 
Tabela 1. Estudos selecionados.

\begin{tabular}{|c|c|c|c|}
\hline Título & Objetivos & Periódico/Ano & Características \\
\hline MELD and other predictors & Examinar quão confiável & Clin Transplant & $(n=436) ;$ AUC ou estatística-C $(0,60)$ \\
\hline of survival after liver & é o sistema de pontuação & 2009 & Baixo poder discriminatório entre o \\
\hline transplantation. Brandão & MELD na previsão da & & MELD e a sobrevida pós-TH. \\
\hline et al. ${ }^{13}$ & sobrevida pós-transplante. & & \\
\hline The impact of the model for & Comparar os resultados do & Arq Gastroenterol & A sobrevida em humano foi \\
\hline end-stage liver disease (MELD) & transplante hepático na era & 2010 & estatisticamente igual na era MELD e \\
\hline on liver transplantation in one & pré-MELD com a era MELD. & & na era pré-MELD: $74,6 \%$ era pré-MELD \\
\hline center in Brazil. Freitas et al..$^{14}$ & & & e $70,9 \%$ na era MELD. \\
\hline Emprego do escore & Analisar a acurácia & Rev Col Bras Cir & A sobrevivência cumulativa em 12 e 24 \\
\hline MELD para a predição & geral do escore MELD & 2012 & meses dos 208 pacientes estudados foi, \\
\hline da sobrevivência pós- & pré-operatório para a & & respectivamente, de $74,5 \%$ e $71,1 \%$. O \\
\hline transplante hepático. & predição da sobrevivência & & escore MELD pré-operatório apresentou \\
\hline \multirow[t]{5}{*}{ Batista et al. ${ }^{15}$} & pós-transplante hepático & & baixo poder discriminatório para a \\
\hline & (pós-TH) e explorar fatores & & predição da sobrevivência pós-TH. Para o \\
\hline & preditivos da sobrevivência & & estrato $\mathrm{CHC}$, o valor correspondente de \\
\hline & de médio prazo (24 meses). & & AUC para a predição da sobrevivência de \\
\hline & & & 24 meses foi de 0,59. \\
\hline Impact of model for end- & Relatar a sobrevida pós- & Transplant Proc & A sobrevida global em dez anos foi \\
\hline stage liver disease score on & transplante hepático de & 2012 & de $67 \%$ ( $n=71)$. A implantação do \\
\hline long-term survival following & pacientes portadores de & & escore MELD teve pequeno impacto \\
\hline liver transplantation for & CHC no Brasil por um & & na sobrevida no longo prazo após o \\
\hline hepatocellular carcinoma. & período de dez anos. & & transplante para o $\mathrm{CHC}$. \\
\hline \multicolumn{4}{|l|}{ Roma et al. ${ }^{16}$} \\
\hline Impact of MELD score & Avaliar o impacto do & Ann Hepatol & Não houve correlação significativa entre os \\
\hline implementation on liver & sistema de pontuação & 2013 & escores de MELD no TH e a sobrevivência \\
\hline allocation: experience at & MELD na alocação e & & pós-TH. Durante o seguimento de 18 \\
\hline a Brazilian center. da Silva & mortalidade hepática após & & meses, a taxa de mortalidade pós-TH \\
\hline \multirow[t]{2}{*}{ Machado et al. ${ }^{17}$} & transplante de fígado no & & foi de $25,4 \%$ antes e $20 \%$ após a \\
\hline & sul do Brasil. & & implantação do MELD (não significativa). \\
\hline Model for End-Stage Liver & Comparar o desempenho & Clinics 2015 & $(n=164)$ pacientes com CHC (16,3\%). \\
\hline Disease, Model for Liver & do escore MELD, entre & & Os achados foram semelhantes aos de \\
\hline Transplantation Survival and & outros, no prognóstico & & Nagler et al. (2005), que obtiveram uma \\
\hline Donor Risk Index as predictive & de sobrevivência pós- & & estatística-C para o MELD de 0,61 e \\
\hline models of survival after liver & transplante hepático. & & mostraram relação entre o escore MELD \\
\hline transplantation in 1,006 & & & e a sobrevida pós-transplante. \\
\hline patients. Aranzana et al. ${ }^{18}$ & & & \\
\hline
\end{tabular}


O primeiro estudo conduzido nos EUA avaliou a sobrevida pós-TH de longo prazo em 1.472 pacientes. Os resultados demonstraram que um MELD pré-TH de 26 foi associado à pior sobrevida no curto e longo prazo (10 anos) pós-TH ${ }^{19}$. Estudos realizados no Brasil, como os de Brandão et al. ${ }^{13} \mathrm{e}$ Batista et al. ${ }^{15}$, utilizaram a análise da área sob a curva ROC (Area under ROC curve, AUC) como medidaresumo do desempenho do escore MELD para a predição da sobrevivência pós-TH. A área sob a curva ROC (Receiver Operating Characteristic), também denominada estatística- $C$, mede a qualidade das previsões de um modelo, com valores a partir de 0,7, considerados clinicamente úteis, enquanto valores iguais ou inferiores a 0,5 relacionam-se a escores com baixo poder discriminatório ${ }^{13,20,21}$. Em ambos os estudos, os pesquisadores encontraram um baixo poder discriminatório (respectivamente, AUC de 0,60 e 0,59) entre o escore MELD e a sobrevida pós-TH.

Freitas et al. ${ }^{14}$, em um estudo comparativo entre os resultados do transplante hepático na era pré-MELD versus a era MELD, observaram que a sobrevida em um ano não obteve diferenças estatisticamente significantes entre os períodos estudados: $74,6 \%$ versus 70,9\%, respectivamente. da Silva Machado et al. ${ }^{17}$ relataram que, após um seguimento de 18 meses, a taxa de mortalidade pós-TH foi de 25,4\% antes e $20 \%$ após a implantação do MELD (resultados estatisticamente não significativos), concluindo que a implantação do escore MELD teve um pequeno impacto na sobrevida em longo prazo de portadores de CHC.

Aranzana et al. ${ }^{18}$ compararam em uma coorte de 164 pacientes portadores de CHC (16,3\%) as áreas sob a curva ROC para sobrevida póstransplante para o escore MELD em um ano e dois anos após o transplante. As áreas sob a curva (AUC) para todos esses intervalos de tempo indicaram que as previsões baseadas no MELD foram mais precisas do que o acaso (ou seja, maior do que 0,5 ).
Há que se ressaltar que o estudo realizado no Brasil foi o único a apresentar relação entre o valor MELD e a sobrevida pós-TH.

Kamath et al. ${ }^{22}$ aduziram que o modelo MELD não tem acurácia em prever a sobrevida em $15 \%$ a $20 \%$ dos casos. No entanto, a porcentagem não se refere exclusivamente aos portadores de CHC. Em relação à capacidade preditiva em médio e longo prazo, estudos brasileiros encontraram um baixo poder discriminatório do escore com a sobrevida pós-TH¹3,15,16.

Nos EUA, Reino Unido e Irlanda, o modelo prognóstico MELD se encontra significantemente associado à sobrevida pós-TH, porém com baixa capacidade preditiva em médio e longo prazo, sugerindo uma combinação do escore MELD com um modelo de sobrevida pós-transplante a ser devidamente desenvolvido e validado 4 .

Em que pese o consenso entre pesquisadores sobre a premência da análise do valor preditivo do escore MELD pós-TH ${ }^{6,10}$, observou-se na literatura a carência de artigos sobre o papel deste modelo na sobrevida pós-TH de médio e longo prazo em portadores de $\mathrm{CHC}$.

\section{CONCLUSÕES}

Embora o número reduzido de artigos elegíveis tenha sido um fator limitante do estudo, nossos resultados corroboraram parcialmente aos encontrados nos EUA, Reino Unido e Irlanda. Nestes países, ao contrário do Brasil, o modelo prognóstico MELD mostrou forte associação com a sobrevida pós-TH. No entanto, a baixa capacidade preditiva do modelo no médio e longo prazo, foi similar ao nosso estudo.

Configura-se a premência do desenvolvimento e validação de um modelo de sobrevida póstransplante para portadores de $\mathrm{CHC}$, para aperfeiçoar o sistema de alocação de órgãos no Brasil. 


\title{
A B S T R A C T
}

\begin{abstract}
This study aimed to analyse the predictive value of Model For End-Stage Liver Disease (MELD) score on medium- and long-term survival in transplanted hepatocellular carcinoma (HCC) patients in Brazil. The study was registered with International Prospective Register of Systematic Reviews (PROSPERO) under N\# 152,363. Inclusion criteria were based on Preferred Reporting Items for Systematic Reviews and Meta-analyses (PRISMA) recommendations. The search was performed on the indexed databases of Lilacs, SciELO, PubMed, and Cochrane Library, and used as search strategy the following Medical Subject Headings (MeSH) terms: ((("MELD Score") OR "Model For End-Stage Liver Disease") AND "Hepatocellular Carcinoma") AND ("Brazil"). We included full-text articles published from January 2006 to October 2019. The initial search found 162 articles. After reading the available abstracts and full texts, 156 articles were excluded, totaling six articles for qualitative analysis. Although the small number of eligible articles was a limiting factor of the study, our results partially corroborated those found in the United States, United Kingdom, and Ireland. In these countries, unlike Brazil, MELD prognostic model has shown a strong association with post-liver transplant (LT) survival. However, the low predictive capacity of the model in medium- and long-term has been similar to the one of our study. The urgency of the development and validation of a post-transplant survival model for patients with HCC is set, improving the organ allocation system in Brazil.
\end{abstract}

Keywords: Liver Transplant. Hepatocellular Carcinoma. Survival Analysis.

\section{REFERÊNCIAS}

1. Salem R, Lewandowski RJ. Chemoembolization and radioembolization for hepatocellular carcinoma. Clin Gastroenterol Hepatol. 2013;11(6):604-11.

2. Ma KW, Cheung TT. Surgical resection of localized hepatocellular carcinoma: patient selection and special consideration. J Hepatocell Carcinoma. 2016;4:1-9.

3. Carrilho FJ, Kikuchi L, Branco F, Goncalves CS, Mattos AA; Brazilian HCC Study Group. Clinical and epidemiological aspects of hepatocellular carcinoma in Brazil. Clinics (Sao Paulo). 2010;65(12):1285-90.

4. Jacob M, Copley LP, Lewsey JD, Gimson A, Toogood GJ, Rela M, Meulen JH; UK and Ireland Liver Transplant Audit. Pretransplant MELD score and post liver transplantation survival in the UK and Ireland. Liver Transpl. 2004;10(7):903-7.

5. Brasil. Ministério da Saúde. Portaria no 1.160, de 29 de maio de 2006. Modifica os critérios de distribuição de fígado de doadores cadáveres para transplante, implantando o critério de gravidade de estado clínico do paciente. Diário Oficial da União, Brasília (DF); 2006 Mai 31.

6. Shiroma RK, Chaib EE, Amed-Filho AM, Ttaniguchi RN, Comarin PR, Handa KK, et al. Transplante de fígado de acordo com os critérios de Milão: revisão dos últimos 10 anos. Rev Med. 2012;91(2):120-4.

7. Sharma $P$, Balan V, Hernandez JL, Harper AM, Edwards EB, Rodriguez-Luna $\mathrm{H}$, et al. Liver transplantation for hepatocellular carcinoma: the MELD impact. Liver Transpl. 2004;10(1):36-41.
8. Yao FY, Bass NM, Ascher NL, Roberts JP. Liver transplantation for hepatocellular carcinoma: lessons from the first year under the Model of EndStage Liver Disease (MELD) organ allocation policy. Liver Transpl. 2004;10(5):621-30.

9. Cholongitas E, Marelli L, Shusang V, Senzolo M, Rolles $K$, Patch $D$, et al. A systematic review of the performance of the model for end-stage liver disease (MELD) in the setting of liver transplantation. Liver Transpl. 2006;12(7):1049-61.

10. Bernardi N, Chedid MF, Grezzana-Filho TJM, Chedid $A D$, Pinto MA, Leipnitz I, et al. Pre-transplant ALBI grade 3 is associated with increased mortality after liver transplantation. Dig Dis Sci. 2019;64(6):1695-704.

11. Moher D, Liberati A, Tetzlaff J, Altman DG; PRISMA Group. Preferred reporting items for systematic reviews and meta-analyses: the PRISMA statement. PloS Med. 2009;6(7):e1000097.

12. NIHR National Institute for Health Research [Internet]. PROSPERO International Prospective Register of Systematic Reviews. Disponível em: https://www.crd.york.ac.uk/prospero/

13. Brandão A, Fuchs SC, Gleisner AL, Marroni C, Zanotelli ML, Cantisani G; Liver Transplantations Group. MELD and other predictors of survival after liver transplantation. Clin Transplant. 2009;23(2):220-7.

14. Freitas AC, Itikawa WM, Kurogi AS, Stadnik LG, Parolin MB, Coelho JC. The impact of the model for end-stage liver disease (MELD) on liver transplantation in one center in Brazil. Arq Gastroenterol. 2010;47(3):233-7. 
15. Batista TP, Sabat BD, de Melo PSV, Miranda LEC, da Fonseca-Neto OCL, Amorim AG, et al. Emprego do escore MELD para a predição da sobrevivência pós-transplante hepático. Rev Col Bras Cir. 2012;39(2):105-11.

16. Roma J, Balbi E, Pacheco-Moreira L, Zyngier I, Araujo A, Agoglia L, et al. Impact of model for end-stage liver disease score on long-term survival following liver transplantation for hepatocellular carcinoma. Transplant Proc. 2012;44(8):2423-7.

17. da Silva Machado AG, de Medeiros Fleck A Jr, Marroni C, Zanotelli ML, Cantisani G, de Melo Brandão AB. Impact of MELD score implementation on liver allocation: experience at a Brazilian center. Ann Hepatol. 2013;12(3):440-7.

18. Aranzana EM, Coppini AZ, Ribeiro MA, Massarollo PC, Szutan LA, Ferreira FG. Model for end-stage liver disease, model for liver transplantation survival and donor risk index as predictive models of survival after liver transplantation in 1,006 patients. Clinics (Sao Paulo). 2015;70(6):413-8.

19. Habib S, Berk B, Chang CC, Demetris AJ, Fontes P, Dvorchik I, et al. MELD and prediction of postliver transplantation survival. Liver Transplant. 2006;12(3):440-7.
20. Xiao L, Fu ZR, Ding GS, Fu H, Ni ZJ, Wang ZX, et al. Prediction of survival after liver transplantation for chronic severe hepatitis B based on preoperative prognostic scores: a single center's experience in China. World J Surg. 2009;33(11):2420-6.

21. Sempere L, Palazón JM, Sánchez-Payá J, Pascual S, de Madaria E, Poveda MJ, et al. Assessing the shortand long-term prognosis of patients with cirrhosis and acute variceal bleeding. Rev Esp Enferm Dig. 2009;101(4):236-48.

22. Kamath PS, Kim WR; Advanced Liver Disease Study Group. The model for end-stage liver disease (MELD). Hepatology. 2007;45(3):797-805.

Recebido em: 23/10/2019

Aceito para publicação em: 12/11/2019

Conflito de interesse: nenhum.

Fonte de financiamento: nenhuma.

\section{Endereço para correspondência:}

Marcel Vasconcellos

E-mail: marcelvasconcellos@unifeso.edu.br marcelvet57@gmail.com

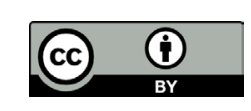

